\title{
Heat Treatment Increases the Level of AGEs in Human Blood
}

\author{
Masako Nakano, Ryoji Nagai
}

Department of Food and Nutrition, Laboratory of Biochemistry and Nutritional Science, Japan Women's University

Levels of plasma pentosidine, which is one of the advanced glycation end-products (AGEs) of the Maillard reaction, are elevated by the pathogenesis of diabetes ${ }^{1)}$ and end-stage renal failure ${ }^{2)}$. A recent study suggested that because plasma pentosidine levels in patients with mild renal dysfunction increase before plasma creatinine levels increase, the measurement of plasma pentosidine level can be a useful clinical marker for the early diagnosis of beginning renal failure ${ }^{3)}$. Several medical laboratories

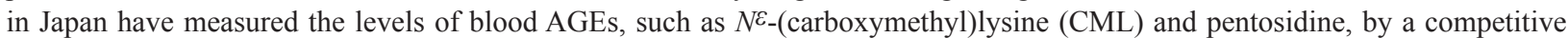
enzyme-linked immunosorbent assay (ELISA). According to the authors' protocol, plasma samples are incubated with proteases for anti-AGE antibodies to assess the intramolecular AGE structures, and are incubated again at $100^{\circ} \mathrm{C}$ for 15 min $^{3)}$ to inactivate the protease. However, our previous study demonstrated that CML, a major antigenic AGE structure, is generated from the Amadori products by short-term heating in vitro, demonstrating that CML generated from Amadori products are an artifact of immunochemical detection by heating process ${ }^{4)}$. Furthermore, Miyata et al. ${ }^{5)}$ reported that substance(s) with a molecular weight of less than 5,000 Da that are abundantly present in uremic plasma exhibit enhanced pentosidine formation during in vitro incubation of uremic plasma at $37^{\circ} \mathrm{C}$, thus strongly demonstrating that pentosidine is also generated by incubation in vitro. To confirm this notion, we conducted additional experiments to clarify whether the pentosidine content measured by high-performance liquid chromatography (HPLC) ${ }^{6}$ in blood increases using the same procedure as that used by medical laboratories. As a result, the levels of pentosidine in patients with nondiabetic hemodialysis increased 1.2- to 3-fold by heating at $100^{\circ} \mathrm{C}$ for 15 min compared to unheated samples. Moreover, the increased rate of pentosidine levels generated by the heating process was found to differ between patients. Therefore, the heating process as a pretreatment for pentosidine measurement by competitive ELISA appears to compromise the accuracy of the pentosidine concentration present in plasma. Taken together, previous reports ${ }^{4,5)}$ and the present study indicate that the level of pentosidine in clinical samples is overestimated by using a heating process. Heating enhances both the oxidative cleavage of Amadori products and the production of $\alpha$-oxoaldehydes such as glucosone, 3-deoxyglucosone, glyoxal, and methylglyoxal, which were indicated to be important precursors for AGE formation ${ }^{4)}$.

We next measured the inhibitory effects of reduction of Amadori products on CML formation. Because CML is generated from the Amadori compounds by oxidative cleavage, whereas the Amadori compounds reduced into hexitol lysine and glucitol lysine are stable and do not easily generate $\mathrm{CML}$, sodium cyanoborohydride $\left(\mathrm{NaBH}_{3} \mathrm{CN}\right)$ is used for the blood CML analysis using a competitive ELISA ${ }^{7}$ to prevent CML formation during the heating process. However, the present study demonstrated that preincubation of glycated bovine serum albumin (glycated BSA), a model Amadori proteins, in the presence of $100 \mathrm{mM}$ sodium borohydride $\left(\mathrm{NaBH}_{4}\right)$, a stronger reducing agent than $\mathrm{NaBH}_{3} \mathrm{CN}$, for $1 \mathrm{~h}$ did not inhibit CML formation induced by heating at $100^{\circ} \mathrm{C}$ for $15 \mathrm{~min}$. From these data, it is likely that the conventional protocol used by medical laboratories which measures plasma pentosidine and CML after pretreatment may be in fact quantifying artifacts of heat treatment. Therefore, the establishment of a more reliable method for the quantification of AGEs in patient blood samples will clarify the physiological significance as well as the clinical usefulness of AGEs. 


\section{References}

1) Sell DR, Monnier VM: End-stage renal diabetes catalyze the formation of pentosidine-derived crosslink from aging human collagen. J Clin Invest 85;380-384:1990

2) Miyata T, Ueda Y, Shinzato T, et al: Accumulation of albumin-linked and free-form pentosidine in the circulation of uremic patients with end-stage renal failure: renal implications in the pathophysiology of pentosidine. J Am Soc Nephrol 7;1198-206:1996

3) Sanaka T, Funaki T, Tanaka $T$, et al: Plasma pentosidine levels measured by a newly developed method using ELISA in patients with chronic renal failure. Nephron 91;64-73:2002

4) Hayashi CM, Nagai R, Miyazaki K, et al: Conver rsion of amadori products of the maillard reaction to $N \varepsilon$-(carboxymethyl)lysine by short-term heating: Possible detection of artifacts by immunohistochemistry. Lab Invest 82;795-808:2002
5) Miyata T, Ueda Y, Yamada Y, et al: Accumulation of carbonyls accelerates the formation of pentosidine, an advanced glycation end product: carbonyl stress in uremia. J Am Soc Nephrol 9;2349-2356:1998 Yoshihara K, Nakamura K, Kanai M, et al: Determination of urinary

6) and serum pentosidine and its application to elder patients. Biol Pharm Bull 21;1005-1008:1998

Yonei Y, Miyazaki R, Takahashi Y, et al: Anti-glycation effect of

7) mixed herbal extract in individuals with pre-diabetes mellitus: a double-blind, placebo-controlled, parallel group study. Anti-Aging Medicine 7;26-35:2010 